\title{
Diferenças de gênero no acesso aos serviços de saúde: problematizações necessárias
}

\author{
Andressa Botton* \\ Sabrina Daiana Cúnico** \\ Marlene Neves Strey***
}

\begin{abstract}
Resumo
O artigo aborda, através de revisão de literatura, a relação histórica entre o (auto) cuidado e a saúde feminina, mais especificamente a saúde sexual e reprodutiva das mulheres, que naturalizou a prática do (auto) cuidado vinculada à identidade feminina. Os homens, porém, são patriarcalmente educados para serem avessos ao cuidado, pois buscar serviços de saúde poderia ser contrário à virilidade, força e independência, características que reforçam e perpetuam a imagem de homem com "H maiúsculo". As estatísticas que demonstram que a expectativa de vida dos homens é mais baixa que a das mulheres parecem se relacionar a essa dificuldade masculina em procurar serviços que contemplem a sua saúde, além da questão sexual e reprodutiva. Levando em consideração as particularidades de homens e mulheres ao praticar o (auto)cuidado, problematiza-se a necessidade de ações de prevenção e promoção de saúde que atinjam a ambos igualmente, possibilitando o incentivo a indivíduos mais saudáveis.
\end{abstract}

Palavras-chave: gênero; saúde; autocuidado.

\section{Gender differences in the access to health services: necesssary problematization}

\begin{abstract}
This article addresses, through literature review, the historical relation between (self)care and female's health, more specifically women's sexual and reproductive health, which naturalized the practice of (self)care linked to the female's identity. However, men are still being educated in an ancient patriarchal way, loath to care, because looking for health services presumably could be seen as opposed to their virility, power and independence, features that reinforce and perpetuate the image of "Macho Man". The statistics which demonstrate that men's life expectancy is lower than women's, it seems to be related to this male difficulty on looking for health services, which contemplate their health beyond sexual and reproductive issues. Taking into consideration men and women's particularities while practicing (self)care, we discuss over the need of health prevention and promotion actions that equally reach both men and women, in order to produce healthies individuals.
\end{abstract}

Keywords: gender, health, selfcare.

* Psicóloga, Mestre e Doutoranda em Psicologia na Pontifícia Universidade Católica do Rio Grande do Sul (PUCRS). Psicóloga na Universidade Federal de Rio Grande do Sul (UFRGS)

** Psicóloga, Mestre em Psicologia (UFSM), Doutoranda em Psicologia pela Pontifícia Universidade Católica do Rio Grande do Sul (PUCRS)

*** Psicóloga, Mestre, Doutora e Pós-Doutora em Psicologia. Professora titular na Faculdade de Psicologia da Pontifícia Universidade Católica do Rio Grande do Sul (PUC-RS), desenvolvendo atividades na graduação e na pós-graduação. Pesquisadora CNPq. Coordenadora do Grupo de Pesquisa Relações de Gênero, da PUCRS. Coordenadora da série Gênero e Contemporaneidade, editada pela EDIPUCRS. 


\section{Introdução}

Historicamente, o cuidado com a saúde e com a vida teve como foco de intervenção as crianças e as mulheres - no que tange ao seu aspecto reprodutivo não tendo se organizado de modo a receber e acolher as demandas masculinas nem as demandas femininas fora da reprodução. A organização do cuidado em torno do binômio mãe-bebê é resultado de um processo histórico de vinculação das ideias médicas com ações políticas voltadas ao corpo feminino. Um corpo visto, prioritariamente, a partir da sua capacidade de gestação e não por meio do reconhecimento da mulher como um sujeito de direitos, necessidades e individualidade (Machin, Couto, Silva, Schraiber, Gomes, Figueiredo, Valença \& Pinheiro, 2011). Os homens, por outro lado, crescem influenciados por uma noção de masculinidade que exige uma eterna vigilância dos gestos, das emoções e do próprio corpo (DaMatta, 2010), o que produz reflexos no campo da saúde, principalmente no tocante à promoção de medidas preventivas (Gomes, 2003).

Essas diferenças, que respondem a uma trajetória histórica e cultural de formatação sobre modos de ser de viver e, também, de se cuidar como homens, ou como mulheres, tem relação direta com as questões de gênero e a forma desigual na qual são transformadas as diferenças entre o gênero masculino e o gênero feminino. Seja porque a orientação para o cuidado de si e dos outros não faz parte da socialização dos homens, ou porque o setor saúde entende a mulher e seu filho como uma díade inseparável, o fato é que as ações em saúde têm sido pautadas em representações conservadoras e aprisionantes. Essas desigualdades com que são tratados/as homens e mulheres acabam, inúmeras vezes, por colocar em risco a saúde desses sujeitos, pois os programas e políticas de saúde ainda privilegiam cuidados estereotipados para cada um dos sexos.

Tal fato nos leva a refletir sobre a necessidade dos programas e políticas de saúde irem além do que vêm estereotipadamente propondo como, por exemplo, para as mulheres, o foco nos cuidados e na preparação para a maternidade (Araújo, 2015; Negrão, 2015). As políticas de saúde atuais negam as potencialidades de transversalizar as questões de gênero em seus programas, o que pode levar a diagnósticos equivocados, ou não considerados, pela prevalência real da doença para o sexo oposto - o que, obviamente, não inviabiliza que acometa ambos os sexos (Strey \& Pulcherio, 2010), como o caso do câncer de mama, que também acomete homens, embora em menor número.
Nessa perspectiva, os estereótipos de "sexo frágil", contrário aos do homem "forte", com "H maiúsculo", vão produzindo práticas de autocuidado intenso para mulheres desde muito cedo, já que seus corpos são vistos como vulneráveis - e futuros reprodutores. Aos homens, ocorre que deleguem o cuidado com sua saúde para as naturalizadas cuidadoras - as mulheres. Essa falta de cuidado masculino com sua própria saúde pode ser decorrência da negligência dos sinais e sintomas, e/ou desconhecimento da - também - fragilidade do próprio corpo, ou ainda pela perpetuação dos estereótipos de gênero de força e virilidade. Um conjunto de fatores que leva a detecção de doenças em um nível muito avançado e a falecimentos prematuros, confirmando o que traz a literatura: que a expectativa de vida masculina, no Brasil, é consideravelmente menor que a feminina (Strey \& Pulcherio, 2010).

Entende-se, assim, a necessidade de olharmos para a temática do cuidado e da saúde de homens e mulheres, além do que habitualmente tem sido feito e que vem reproduzindo estereótipos de gênero de forma tradicional e acrítica. Considerando tais aspectos, este artigo tem por objetivo problematizar e refletir sobre as relações de gênero nas ações de prevenção e promoção de saúde, considerando as diferentes demandas de homens e mulheres da atualidade e o alcance que tais ações têm em sua saúde sexual e reprodutiva. Especificamente, objetiva-se, analisar como o (auto)cuidado com a saúde sexual e reprodutiva é entendido e praticado por homens e mulheres contemporâneos/as, bem como refletir sobre os estereótipos de gênero nas ações de prevenção e promoção de saúde para homens e mulheres na atualidade.

\section{Método}

Para atingir os objetivos propostos, faz-se necessário a revisão de literatura que reúne materiais já publicados sobre determinado assunto para avaliá-los criticamente, contemplando a temática escolhida (APA, 2010). Esse método é uma revisão do estado da arte do tema, o qual permite identificar como e onde eles estão disponíveis, no âmbito acadêmico-científico (Hohendorff, 2014).

Para organizar a pesquisa, buscou-se publicações em livros e artigos científicos que abordam as temáticas principais de gênero, práticas de saúde e (auto)cuidado, prevenção e promoção de saúde a homens e mulheres e outras inter-relacionadas. Delimitou-se a pesquisa com publicações dos últimos quinze anos dando ênfase, em especial, àquelas que contemplam produções com o viés problematizador, bem como trazem autores e autoras referências no tema. 


\section{Resultados e discussão}

\section{Os homens e a prática do autocuidado: uma associação possível?}

No que tange ao reconhecimento dos homens como sujeitos de cuidado e como potenciais cuidadores, atentamos para a invisibilidade deste reconhecimento na agenda do setor de saúde brasileiro. Isto porque, os homens aparecem nesta agenda prioritariamente como parceiros das mulheres na atenção à reprodução, na responsabilização pelo aumento da contaminação por HIV nos casais heterossexuais e, mais recentemente, pela violência perpetrada contra as mulheres (Machin et al., 2011; Schraiber, Gomes \& Couto, 2005). Nesse sentido, os homens são, inúmeras vezes, apresentados nos discursos das campanhas de saúde, não devido à preocupação com a sua saúde em si, mas como relativo a problemas que causam aos outros - no caso às mulheres.

Esse fato reforça a invisibilidade masculina e o quanto as campanhas de promoção e prevenção à saúde, embora tenham como objetivo formal atingir os homens, trazem a questão do cuidado para a esfera feminina, reforçando os estereótipos de que esse é o sexo que precisa de maiores cuidados (Kohn \& Strey, 2011). Além disso, responsabilizam os homens por práticas sexuais de risco que, embora precisemos falar sobre a importância dessa prevenção, não são exclusivas dos homens (Machin et al, 2011).

Discursos como esses, reiteram que as mulheres não podem - e que, de certa forma, que não devem ter esses comportamentos, já que o usufruto do corpo vinculado à prática sexual pelo prazer ainda está ligado, no imaginário social, ao masculino. Campanhas que promovem tais ideias não contemplam de forma plena uma prevenção às doenças e mortes precoces e evitáveis, bem como perpetuam estereótipos tradicionais sobre os homens e as mulheres.

Vale lembrar que foi a constatação de que os homens possuem uma esperança de vida mais baixa, bem como taxas de mortalidade mais altas no comparativo às mulheres, que impulsionou a criação pelo Ministério da Saúde, no Brasil, em 2008, da Política Nacional de Atenção Integral a Saúde do Homem (PNAISH). Tal política tem o objetivo de promover ações de saúde para a população masculina de forma integral, a fim de reduzir os índices de morbimortalidade por causas possíveis de prevenir e evitáveis e aumentar a expectativa de vida nessa população (Brasil, 2008). No entanto, a consolidação e mobilização desta política é, muitas vezes, impossibilitada pela existência de crenças sociais que fazem com que os homens não procurem os serviços de saúde.

Estas crenças sociais reproduzem um ideal de masculinidade que vincula aspectos como a virilidade e a invulnerabilidade com a crença de um homem com "H maiúsculo", impedindo que os homens adotem práticas de autocuidado, por exemplo. Procurar os serviços de saúde numa perspectiva preventiva poderia, a partir dessa lógica, revelar a fraqueza, o medo e a insegurança dos homens, aproximando-os das representações do universo feminino (Gomes, Nascimento \& Araújo, 2007).

Em pesquisa realizada, cujo objetivo foi analisar os discursos dos usuários que - não - são beneficiados pela PNAISH, e dos profissionais que fazem os atendimentos, os dados revelam que a busca pelo cuidado e tratamento ocorre quando os sintomas e doenças já avançaram bastante, culminando em um diagnóstico de maior gravidade, com maior taxa de mortalidade. Uma hipótese para a resistência em buscar auxílio médico seria a dificuldade de compreenderem a necessidade da prevenção, já que um corpo forte e viril, segundo os estereótipos de gênero, nunca adoeceria. Além disso, as pesquisadoras citam a falta de autonomia dos homens em relação a sua saúde, ficando essa prática vinculada ao cuidado que as mulheres de suas vidas (mães, esposas ou companheiras, filhas, irmãs) têm com eles (Kohn \& Strey, 2011).

Neste panorama, em que aspectos como a sensibilidade, o cuidado do outro e de si e a fragilidade são vistas como características femininas, os homens se distanciam de tais características, passando a exprimir comportamentos de risco que os predispõem a doenças, lesões e morte (Korin, 2001; Schraiber, Gomes \& Couto, 2005). Como exemplo desta afirmação, podemos citar a prevenção do câncer de próstata, pois há, sobre a saúde sexual e reprodutiva masculina, uma ambiguidade. Quando estão com algum sintoma, os homens têm interesse em buscar ajuda médica para não perderem suas parceiras, por exemplo, por não conseguirem praticar atividade sexual. Ao mesmo tempo, têm preconceito e resistência em fazer o exame de toque retal, realizado por profissionais de medicina como uma das medidas preventivas deste tipo de câncer (Kohn \& Strey, 2011). Em função deste procedimento supostamente ameaçar o status de macho e a inviolabilidade masculina, muitos homens optam por não realizá-lo, aumentando as taxas de incidência e de mortalidade resultante deste tipo de câncer (Brasil, 2008).

DaMatta (2010), ao problematizar o receio de alguns homens em se submeter a este procedimento, afirma que é a parte da frente - o falo - que diferencia o homem da 
mulher. A nádega, por outro lado, os igualam, se constituindo como a parte mais feminina do corpo masculino. Realizar o toque retal, portanto, suscita nos homens "O medo de ser tocado na sua parte "inferior"' (Gomes, 2003, p. 828), sendo que tal medo se desdobra em inúmeros outros, como o medo da dor, de ter ereção e/ou de ficar descontraído e isso ser interpretado como sinal de que o toque é algo comum e prazeroso (Gomes, 2003).

Temos, assim, um círculo vicioso que invisibiliza e, também, exclui os homens dos discursos de promoção e prevenção da saúde. Além de não serem educados para a prática do autocuidado, os serviços de atenção primária são despreparados para receberem os homens. Isto porque, muitos/as trabalhadores/as da saúde trazem consigo modos de pensar, sentir e agir que são provenientes dos valores e das crenças do que é ser homem numa cultura machista. Tais julgamentos e comportamentos estereotipados se opõem às práticas de cuidado humanizado que são normativas do Sistema Único de Saúde (SUS), uma vez que podem agravar as desigualdades de saúde, na medida em que propõem atendimentos inadequados por falta de uma escuta qualificada e de um acolhimento que dê conta da diversidade dos homens e dos estilos de masculinidades.

Soma-se a isto o fato de que a implantação do PNAISH nos municípios brasileiros é escassa (Figueiredo \& Schraiber, 2011) e pela baixa procura os/as profissionais e a abordagem da equipe é mais bem treinada para atender mulheres, crianças e idosos. Inclusive, até a decoração desses espaços de acolhimento é mais voltada ao público feminino, bem como as abordagens das campanhas e das estratégias de intervenção, prevenção e promoção de saúde (Couto, Pinheiro, Valença, Machin, Silva, Gomes, Schraiber \& Figueiredo, 2010). Assim, os lugares, discursos e profissionais que deveriam cuidar da saúde dos homens, por inúmeros fatores, estão distantes da realidade desejada, que contribui para um aumento nos números de doenças e mortes masculinas, como já citado.

\section{As mulheres e o autocuidado: espaços possíveis para além da maternidade}

"Este nosso corpo sempre teve muito pouco de nosso, foi muito mais o corpo que alguns gostariam que fosse" (Colling, 2004, p. 49). Essa frase resume a ideia defendida pela autora de que o corpo feminino foi historicamente constituído como inferior ao masculino. Isso reflete a necessidade de cuidado ensinados as mulheres desde o momento que nascem. O corpo feminino é dito frágil - afinal, nós somos o "sexo frágil" - e, então, precisa de mais (auto)cuidado, mais atenção e mais saúde.
Essa aura da necessidade de cuidado, quando vista com olhos críticos, aponta para os discursos estereotipados das ações de promoção e prevenção de saúde. As políticas de saúde e (auto)cuidado voltadas para as mulheres têm como foco a saúde sexual e reprodutiva, tendo "um olhar em direção à mulher no seu papel de reprodutora e responsável por todo o processo reprodutivo desde o antes até o depois, ou seja, a prole" (Taquette, 2012, p. 85-86).

A fragilidade imposta às mulheres traz o aprendizado do autocuidado muito mais presente que na vida dos homens. Atualmente é comum que, desde a pré-adolescência, as meninas já sejam incentivadas aos exames ginecológicos preventivos, pois - mesmo sem ser questionadas - já se presume que o corpo feminino, em algum momento, abrigará um novo ser e precisa estar saudável e preparado para isso.

Assim, fala-se na "nova politização da maternidade", a qual apresenta a uma mulher que pretende engravidar, ou que já está grávida, diferentes pedagogias do cuidado de si e do outro (do feto). Trata-se de uma série de cuidados corporais, cada vez mais complexos e com mais investimentos: cuidado pré-natal (e anterior à gestação), cursos especializados, esportes e alimentos adequados. $\mathrm{Na}$ atualidade, as gestantes são incentivadas a praticar a ideia de cuidar de si, cuidar da vida, estreitando a percepção em relação ao próprio corpo com disciplina, autodomínio e vigilância. As grávidas são cobradas para aperfeiçoarem e corrigirem seus corpos, ao mesmo tempo que desenvolvem as competências que as permitirão cuidarem de outra vida (Schwengber \& Meyer, 2015).

Tal fato ainda é um balizador das campanhas de saúde da mulher, mostrando a necessidade de problematização e mudança nesse contexto. No Brasil é tema de saúde pública e envolve diferentes dispositivos de informação, como os meios de comunicação, o governo e os serviços de saúde, que colocam a maternidade como uma condição essencial ao sexo feminino. Esses orientam para as práticas de um ideal de mulher-mãe tradicional em relação aos estereótipos de gênero (Cadoná \& Strey, 2014).

Nessa perspectiva, há setores da saúde da mulher que ficam invisibilizados e, consequentemente, negligenciados. É o caso das demandas relacionadas ao sofrimento pela jornada dupla/tripla, à violência doméstica, à saúde psíquica e à prática da sexualidade pelo prazer, desvinculado do objetivo da reprodução. Então, para onde se dirigem essas mulheres que desejam usufruir saudavelmente da potencialidade de seus corpos? (Villela \& Monteiro, 2005). 
É possível dizer que muitas vezes, quando fogem do padrão de mulher-mãe, mulher-esposa, mulhermonogâmica e outros diferentes estereótipos de gênero, ficam marginalizadas aos discursos que deveriam proteger sua saúde. Qualquer especificidade ou subjetividade que subverta o esperado, parece escapar das preocupações de espaços públicos e privados com a saúde feminina.

Nessa intenção, o Ministério da Saúde lançou em 2013 a cartilha "Mulheres Lésbicas e Bissexuais: Direitos, Saúde e Participação Social". O intuito foi promover a visibilidade desse público como usuárias dos espaços de saúde e sensibilizar e orientar profissionais de saúde para um acolhimento adequado, sem preconceito, discriminação ou imposição de expectativas que fazem parte da vida do(a) profissional, mas não da paciente. (Brasil, 2013)

Salienta-se a importância dessa estratégia para a discussão das questões da saúde feminina para além dos estereótipos de gênero, permitindo a circulação desses discursos, bem como aqueles para além do binário, como nos casos das transexuais, travestis e transgêneros. Essas/ Esses inúmeras vezes, não encontram acolhimento adequado e humano em espaços que deveriam cuidar e orientar sobre sua saúde de forma integral, o que prejudica o usufruto de seu corpo e de sua sexualidade (assim como de todos/as que deparam-se com os entraves baseados em preconceitos dos profissionais e serviços de saúde) (Freire, Araújo, Souza \& Marques, 2013).

Uma possibilidade no campo da saúde pública e da cultura brasileira é que o termo "direitos sexuais e reprodutivos", historicamente presente nas políticas públicas direcionadas à mulheres, possa ser utilizado de forma separada, utilizando-se "direitos reprodutivos" para quando deseja-se atingir o cuidado com a vida reprodutiva das mulheres - e dos homens -, e "direitos sexuais", que representaria o exercício da sexualidade pelas mulheres, assim como aos homens já é permitido e incentivado (Sampaio, 2015).

Além disso, sugere-se que tais conceitos sejam colocados no campo do poder e dos recursos, delimitando a reprodução como um direito feminino - e não uma obrigação, e a sexualidade como algo que pode ser livremente praticado, sem qualquer preconceito ou discriminação. É permitir às mulheres e aquelas que se veem e se sentem como tal decidir sobre seus corpos, podendo vivenciar com prazer sua sexualidade, caminhando para a igualdade de gênero referente a esse tema (Sampaio, 2015).

Nesse sentido, cabe ao estado a prevenção e a promoção da saúde de forma universal e igualitária em relação ao gênero nos serviços que são oferecidos à população, primando pela qualidade. Porém, cabe a cada cidadão a conscientização dessa importância, a problematização nos espaços devidos e, consequentemente, a luta pela ampliação do acesso a serviços de qualidade, o que acreditamos ser essencial para o bem-estar cotidiano, respeitando toda e qualquer especificidade nos modos de ser e viver em relação ao gênero.

\section{Considerações finais}

As diferenças entre homens e mulheres sempre existiram e sempre existirão, pois somos biologicamente diferentes. Entretanto, a compreensão das questões de gênero e do feminismo aponta que não devemos deixar que as diferenças biológicas sejam entendidas como desigualdade entre os homens e as mulheres.

Isso porque, apenas o fato de ser homem já garante tratamento superior e denota maior poder em relação às mulheres. Nesse sentido, atentamos que o primeiro passo é entender que tal demonstração de desigualdade, como visto, traz muitos prejuízos à saúde e à vida das mulheres, mas também traz perdas à saúde e à vida dos homens, que não podem sucumbir à qualquer fragilidade, a fim de manter seu status de virilidade (Van Dijk, 2012).

As argumentações aqui tecidas buscam sinalizar o quanto os estereótipos de gênero ainda permeiam a prática do (auto)cuidado e das políticas públicas em saúde, fazendo com que os discursos sobre prevenção e promoção de saúde não atinjam homens e mulheres de modo integral e equivalente. Nesse sentido, faz-se necessário pontuar que, saberes hegemônicos, tal como a saúde pública, devem questionar permanentemente se suas ações estão de acordo com as necessidades da população que pretendem atingir (Franco, 2014). Defende-se, assim, que esse é um questionamento relevante e que necessita ser constante entre os/as profissionais que cuidam da saúde de homens e mulheres.

Reconhecer que homens e mulheres são seres gendrados possibilita a compreensão da dimensão histórica, a qual é altamente influenciada por aprendizagens socioculturais que determinam as regras e as maneiras de cada gênero se posicionar na estrutura social (Franco, 2014). Tomando por base essa definição, problematiza-se a importância da desconstrução dos estereótipos de gênero nos espaços de saúde. Esses que ainda vinculam as necessidades da mulher a uma possível gestação e desconsideram a importância da inserção dos homens nas práticas de (auto)cuidado.

Nessa perspectiva, compreende-se que o reconhecimento das necessidades individuais de homens e mu- 
lheres para além destes estereótipos, poderia possibilitar que os discursos produzidos no âmbito da saúde fossem direcionados para a construção de políticas que atinjam de modo efetivo e integral aos homens e às mulheres. Nessa via, ao especificar-se as práticas do (auto)cuidado para ambos os sexos, podemos produzir espaços de saúde mais acolhedores, profissionais mais qualificados e maior promoção de saúde para aqueles/as que buscam esses espaços.

É urgente e necessário mudarmos o posicionamento das estratégias de prevenção e promoção da saúde, bem como as formas de intervenção enquanto profissionais da saúde. As questões de gênero precisam ser entendidas e praticadas como um conteúdo transversal a todas as temáticas da saúde, e não vinculado a grupos ou a campanhas específicas. O desconhecimento da amplitude das temáticas de gênero está tornando muitas demandas, de homens e de mulheres, invisíveis. E essa invisibilidade vem trazendo a exclusão dos espaços de acolhimento e de saúde e, consequentemente, o adoecimento e a letalidade - o oposto do que propõem tais locais e suas campanhas.

\section{Referências}

American Psychological Association (2010). Publication manual of American Psychological Association (6th ed). American Psychological Association: Washington DC.

Araújo, M. J. O. (2015). Modelos de atenção à saúde mental das mulheres: linhas de cuidado na perspectiva dos direitos humanos, gênero e integralidade na saúde. In Negrão, T.; Vargas, R. \& Rodrigues, L. P. (Orgs.). Saúde Mental e Gênero: novas abordagens para uma linha de cuidado (pp. 55-76). Porto Alegre: Coletivo Feminino Plural.

Brasil. (2008). Ministério da Saúde, Política Nacional de Atenção Integral à Saúde do Homem (Princípios e Diretrizes). Brasília: Secretaria de Atenção à Saúde/ Departamento de Ações Programáticas Estratégicas.

Brasil. (2013). Ministério da Saúde, Mulheres Lésbicas e Bissexuais: Direitos, Saúde e Participação Social. Brasília: Secretaria de Gestão Estratégica e Participativa/ Departamento de Apoio à Gestão Participativa.

Cadoná, E. \& Strey, M. (2014). A produção da maternidade nos discursos de incentivo à amamentação. Estudos Feministas, 22(2), 477-499.

Colling, A. M. (2004). O corpo que os gregos inventaram. In Strey, M. N. \& Cabeda, S. T. L. (Orgs.). Corpos e subjetividades em exercício interdisciplinar (pp. 49-64). Porto Alegre: EDIPUCRS.

Couto, M.T.; Pinheiro, T. F.; Valença, O.; Machin, R.; Silva, G. S. N.; Gomes, R.; Schraiber, L. B.; \& Figueiredo, W. S. (2010). Men in primary healthcare: discussing (in)visibility based on gender perspectives. Interface - Comunicação, Saúde, Educação, 14(33), 257-70.

DaMatta, R. (2010). Tem pente ai? Reflexões sobre a identidade masculina. Enfoques, 9(1), 134-151.
Figueiredo, W. S; \& Schraiber, L. B. (2011). Concepções de gênero de homens usuários e profissionais de saúde de serviços de atenção primária e os possíveis impactos na saúde da população masculina. Ciência e saúde coletiva 16(1), 935-944.

Franco, N. E. M. (2014). El cuidado de sí masculino como tema necessario en los debates contemporáneos de la salud pública. In Strey, M. N; Von Mühlen, B. K; \& Kohn, K. C.(Orgs.). Caminhos de Homens: gênero e movimentos. (pp. 237-260). Porto Alegre: EDIPUCRS.

Freire, E. C.; Araújo, F. C.; Souza, A.; Marques, D. (2013). A clínica em movimento na saúde de TTTS: caminho para materialização do SUS entre travestis, transsexuais e transgêneros. Saúde em debate, 62(4), 477-484.

Gomes, R. (2003). Sexualidade masculina e saúde do homem: proposta para uma discussão. Ciência e saúde coletiva, 8(3), 825-829.

Gomes, R.; Nascimento, E. F.; \& Araújo, F. C. (2007). Por que os homens buscam menos os serviços de saúde do que as mulheres? As explicações de homens com baixa escolaridade e homens com ensino superior. Cadernos de Saúde Pública, 23(3), 565-574.

Hohendorff, J. V. (2014). Como escrever um artigo de revisão de literatura. In Koller, S., Couto, M. C. P. \& Hohendorff, J. V. (Orgs.) Manual de produção cientifica (pp. 39-54). Porto Alegre: Penso.

Kohn, K. C., \& Strey, M. N. (2011). A (nova) política de saúde para homens: abrindo caminhos para os discursos masculinos. Dissertação (Mestrado em Psicologia), Pontifícia Universidade Católica do Rio Grande do Sul, Porto Alegre.

Korin, D. (2001). Novas perspectivas de gênero em saúde. Adolescencia Latinoamericana, 2(2), 67-79.

Machin, R.; Couto, M. T.; Silva, G. S. N.; Schraiber, L. B.; Gomes, R.; Figueiredo, W. S.; Valença, O. A; \& Pinheiro, T. F. (2011). Concepções de gênero, masculinidade e cuidados em saúde: estudo com profissionais de saúde da atenção primária. Ciência \& Saúde Coletiva, 16(11), 4503-4512.

Negrão, T. (2015) Apresentação. In Negrão, T.; Vargas, R. \& Rodrigues, L. P. (Orgs.). Saúde Mental e Gênero: novas abordagens para uma linha de cuidado (pp. 7-12). Porto Alegre: Coletivo Feminino Plural.

Sampaio, P. F. (2015). Direitos sexuais e reprodutivos. In Colling, A. M., \& Tedeschi, L. A. (Orgs.). Dicionário Crítico de Gênero (pp. 166-170). Dourados: UFGD.

Schraiber, L. B., Gomes, R. \& Couto, M. T. (2005). Homens e saúde na pauta da saúde coletiva. Ciência e saúde coletiva, 10(1), 7-17.

Schwengber, M. S., \& Meyer, D. E. Gravidez. (2015). In Colling, A. M. \& Tedeschi, L. A. (Orgs.). Dicionário Crítico de Gênero (pp. 316-319). Dourados: UFGD.

Strey, M. N \& Pulcherio, G. (2010). As tramas de gênero na saúde. In Strey, M. N; Nogueira, C. \& Azambuja, M. P. R. (Orgs.) Gênero e Saúde: diálogos ibero-brasileiros. (pp. 11-33). Porto Alegre: EDIPUCRS.

Taquette, S. (2012). A importância da visão do gênero nas práticas de saúde com adolescentes. In Strey, M. N.; Botton, A.; Cadoná, E.; \& Palma, Y. A. (Orgs). Gênero e ciclos vitais: desafios, problematizações e perspectivas (pp. 67-95). Porto Alegre: EDIPUCRS.

Van Dijk, T. A. (2012). Discurso e poder. São Paulo: Contexto.

Villela, W., \& Monteiro, S. (2005). Atenção à saúde das mulheres: historicizando conceitos e práticas. In Villela, W. \& Monteiro, S. (Orgs.). Gênero e Saúde (pp. 15-32). Rio de Janeiro, ABRASCO.

Submetido em: 1-11-2016

Aceito em:6-3-2017 